\title{
"Praga è solal:: il Sessantotto in Cecoslovacchia raccontato dalla stampa italiana (gennaio 1968 - settembre 1969)
}

\author{
"Praga è sola»: the 1968 in Czechoslovakia, a tale told by \\ the Italian press (January 1968 - September 1969)
}

\author{
Onofrio Bellifemine \\ Università Cardinale Stefan Wyszyński, Polonia
}

\begin{abstract}
Riassunto: La Primavera di Praga è stato uno degli eventi più significativi del 1968, capace di lasciare larghe tracce nella storia del '900 europeo. Analizzando i principali quotidiani e riviste italiane del periodo, questo saggio intende fornire un'interpretazione delle linee di lettura, delle analisi e delle cronache giornalistiche più interessanti di quell'evento. Particolare attenzione è stata dedicata a tre momenti che hanno avuto una particolare importanza nello svolgimento dell'intera vicenda: la caduta di Novotný e l'ascesa di Dubček; il consolidamento del gruppo riformista; l'intervento armato sovietico e la reazione della stampa italiana.
\end{abstract}

Parole chiave: giornalismo, URSS, Cecoslovacchia, Guerra Fredda, Primavera di Praga, Sessantotto.

\begin{abstract}
Prague's Spring was one of the most significant event of 1968, able of leaving large signs in the history of the 20th century in Europe. This essay intends to provide an interpretation of the most interesting reading lines, analysis and journalistic reports of that event through the main Italian newspapers and magazines of that period. A particular attention is given to three important moments that were the turning point of that event: the falling of Novotný and the rising of Dubček; solidification of the reformist's group; the armed intervention of the soviets and the reaction of the Italian press.
\end{abstract}

Keywords: journalism, USSR, Czechoslovakia, Cold War, Prague Spring, protests of 1968.

\section{Introduzione}

La Primavera di Praga fu un convulso periodo di «lotte ardue, speranze fervide e delusioni amare» (Rozgonovà, 2009: 5) destinato sin dall'inizio a segnare in modo cruciale la storia dell'Europa centro-orientale ma anche a toccare profondamente l'opinione pubblica del mondo occidentale come può testimoniare la straordinaria eco mediatica che quelle vicende suscitarono. In Italia, i fatti praghesi godettero di una copertura giornalistica capillare e costante con resoconti giornalieri, inchieste, ricostruzioni politiche, interviste ad esponenti politici ed intellettuali, dettagliate analisi 
e previsioni di futuri, possibili scenari. L'obbiettivo di questo lavoro è quello di cogliere le linee interpretative di maggior interesse e di analizzare le varie fasi seguite dal racconto giornalistico.

\section{1. «Comunismo con libertà?»: dalla caduta di Novotný all'ascesa di Dubček}

II 5 gennaio del 1968 Antonín Novotný segretario del Partito comunista cecoslovacco, si dimetteva lasciando il suo posto a un ancora poco conosciuto dirigente slovacco, Alexander Dubček. Era un cambiamento rilevante e altamente simbolico che segnava una nuova fase in uno dei paesi comunisti più strategici e si inseriva in una complessa e assai articolata stagione di riforme avviata in Urss da Brèžnev e Kosygin e finalizzata a una moderata liberalizzazione (Graziosi, 2008: 297369; Bottoni, 2011: 186-216; Arbatov, 1992; Ajtmatov, 1982).

Tali provvedimenti, in maniera inaspettata per i loro promotori, impressero nei Paesi dell'Europa Orientale un potente influsso al riformismo, che uscì dal ristretto ambito economico per intaccare il nucleo strutturale degli ordinamenti politici (Zaslavy, 2009: 99).

Il quotidiano socialista Avanti! non aveva dubbi: si trattava di un vero e proprio «terremoto politico» che poneva già dalle sue primissime battute "grossi interrogativi di carattere interno e internazionale») (Avantil, 7 gennaio 1968: 3). Iniziava così per la Cecoslovacchia, un periodo denso e travagliato di riforme, un tentativo di modernizzare e democratizzare il paese, conciliando una precisa appartenenza di campo, quella comunista, con la libertà e la democrazia (Leoncini, 1989; Leoncini 2003; Leoncini 2009; Havel 1991; Laudiero, 2018; Ripellino, 2008; Bettiza, 2009; Volcic, 2008; Guida, 2009; Fedele \& Fornaro, 2009; Bottoni, 2011: 216-223). Toni più sobri, invece, furono utilizzati da l'Unità che dette la notizia del cambio al vertice in Cecoslovacchia con un articolo del giornalista e storico Giuseppe Boffa che faceva presente come la decisione rientrasse unel quadro di una redistribuzione delle massime cariche del paese, imperniata sulla separazione delle due altissime funzioni di presidente della Repubblica e di primo segretario del partiton) (Boffa, 6 gennaio 1968: 1)'. Le rapide trasformazioni politiche che si susseguirono già nei primi giorni di gennaio ponevano immediati e gravi quesiti di difficile soluzione. I principali giornali italiani si interrogavano sulla reale consistenza della trasformazione in atto, su quali binari il nuovo corso riformatore guidato da Dubček si sarebbe mosso, su quali forze all'interno della società cecoslovacca avrebbe fatto affidamento la nuova fase e soprattutto su quale sarebbe stata la reazione di Mosca, se improntata a una tollerante collaborazione o ad una autoritaria reazione. Erano interrogativi che chiaramente ne aprivano di più ampi sulla compatibilità tra comunismo e libertà democratiche e più in generale sulla possibilità del blocco sovietico di riformarsi e modernizzarsi abbracciando istanze pluralistiche. II cambio al vertice che si consumava a Praga in quei primi giorni di gennaio, appariva emblematico: Novotný, a lungo capo del comunismo cecoslovacco e simbolo di una fede politica ortodossa e di stampo conservatore, lasciava il campo a Dubček, un funzionario più giovane, di ideali liberali e progressisti, capace di rappresentare al meglio le istanze di cambiamento chieste a gran voce dalle forze più vitali e dinamiche della società. Il Messaggero offrì una serie di ritratti del vecchio capo comunista che ne mettevano in mostra il duro autoritarismo, la fedeltà a uno schema politico superato

1 Sul Pci e la Primavera di Praga: Hobel, 2001: 1145-1172; Zaslavsky, 2009; Pajetta, 1982. 
e inquietante, quello dello stalinismo, ma anche una indiscussa astuzia strategica, un'accortezza tattica che gli aveva permesso di scalare posizioni su posizioni all'interno della nomenclatura del partito. II 5 gennaio Novotný veniva presentato come un capo stalinista:

[...] che oltre a alimentare il culto della personalità di pretta marca staliniana ha fatto il possibile per frenare le riforme economiche liberaleggianti dei pianificatori del partito portando il Paese praticamente al limite dell'inflazione (II Messaggero, 5 gennaio 1968: 1).

Un vero e proprio «piccolo Machiavelli» che "è sempre riuscito a battere gli avversari ma questa volta è stato messo in minoranza e la rivoluzione di palazzo a Praga contro il tiranno stalinista si è conclusa a suo dannolı. Sullo stesso giornale Alfonso Sterpellone ${ }^{2}$ riconosceva a Novotný buone capacità di organizzatore politico ma nel contempo sottolineava la sua linea di comando dispotica e l'opposizione sempre più agguerrita coltivata da economisti e intellettuali. Con lui tramontava «l'ultimo stalinista del blocco est-europeo che intendeva perpetuare un sistema anacronistico di governo» (Sterpellone, 7 gennaio 1968: 1). Ma il quotidiano romano non leggeva nel cambiamento in atto un'evidente frattura politica facendo notare che il nuovo leader, Dubček, era «un comunista educato in URSS. L'influenza sovietica in Cecoslovacchia continua assoluta»».

Il quotidiano democristiano II Popolo invece dava un'altra lettura della caduta del vecchio leader comunista tradito proprio dalle sue doti di temporeggiatore:

[...] gli osservatori rilevano che Novotný è rimasto vittima delle sue doti di tattico. Per circa quindici anni egli si è mantenuto al potere evitando sempre i confronti diretti, eccellendo nell'arte del compromesso. Stalinista per formazione e temperamento, [...] si impose al presidente Gottwald al quale non era gradito e ne prese la successione (II Popolo, 7 gennaio 1968: 1).

Novotný restava presidente della Repubblica: avrebbe perso anche quell'incarico il successivo 21 marzo, uscendo definitivamente di scena. In quell'occasione Enzo Biagi ${ }^{3}$ su La Stampa ne tratteggiò la personalità in un lungo ritratto, acuto e sottile che cercava di mettere in evidenza soprattutto le caratteristiche in grado di raccontare un'intera epoca. "Onesto e noioso», più «burocrate» che "leader del partito», dell'Urss aveva accettato tutto: "la linea di Stalin e quella di Kruscev, il terrore ed il disgelo», ma adesso «non trova posto in un Paese che sta chiedendo di tornare alle sue tradizioni civilis. Novotný raccontava bene un'intera generazione di dirigenti:
[...] di lui si sapeva ben poco: un'aneddotica senza rilievo, una storia che assomiglia a quella di altri compagni, Kadar e Gomulka, ad esempio [...] c'è sempre all'inizio un padre operaio, e uno sciopero, poi la scoperta del partito e d'una passione che durerà per sempre. Le vicende di questi personaggi hanno tappe d'obbligo: i cortei di dimostranti, le letture disordinate, il carcere, le lotte interne, i campi di concentramento, i gendarmi della reazione, i soldati di Hitler, quelli di Stalin, la conquista del potere, le crisi e le condanne. L'onesto Novotný come dicevano gli

\footnotetext{
2 Alfonso Sterpellone è stato corrispondente da Mosca per il Messaggero e per l'Ansa. Su Sterpellone: Dicorato, 2012: 84 .

3 Per un profilo di Enzo Biagi: http://www.treccani.it/enciclopedia/ricerca/enzo-biagi/ [02/02/2020].
} 
estimatori, è cresciuto nel clima del comunismo di Stalin, e possedeva come tutti i bravi militanti senso di adattamento che permette di accettare in vista della felicità ultima tutti i contorcimenti della politica, le necessità tattiche, la revisione ideologica, ma non poteva nascondere la sua natura, nénascondere la sua ultima vocazione. Ha passato i sessanta, è stanco ma difende con ostinazione il prestigio e il comando. Non lascerà rimpianti ma una penosa eredità. II comunismo in cui credeva ha fomentato ribellioni ed eresie (Biagi, 20 marzo 1968: 3).

Poco invece si sapeva del nuovo gruppo dirigente. L'Avanti! cercava di rispondere a beneficio dei lettori alla domanda su chi fossero gli uomini nuovi di Praga e cosa rappresentassero. Dubček veniva definito riformista ma prudente, alla testa di un gruppo dirigente deciso a democratizzare il paese cercando però allo stesso tempo di recuperare il consenso e le simpatie in chiave filosovietica dell'opinione pubblica che «stava slittando su posizioni filo-occidentali»».

Si trattava di una uimpresa molto difficile» perché comportava il rischio di possibili rotture politiche come quelle che in Ungheria nel 1956 erano culminate «nell'insurrezione di Budapest». Veniva avanzata anche una previsione quantomai profetica: «la tensione rimarrà ancora acuta e le prospettive estremamente incerte» (Avantil, 7 gennaio 1968: 1). Ma la vicenda cecoslovacca non veniva letta unicamente come una schermaglia di potere tra conservatori e riformisti. II Popolo ad esempio forniva un'interpretazione più articolata che andava in due direzioni: da una parte si faceva notare la crisi profondissima del blocco sovietico, con Mosca incapace ormai di evitare lo scivolamento di pezzi importanti del proprio impero come la Cecoslovacchia ma anche la Polonia e la Romania verso le politiche liberali del capitalismo americano dettate da Kiesinger. Dall'altra parte si passava ad analizzare con una certa cura la società cecoslovacca solcata da più linee di frattura e nella quale si stavano ritagliando uno spazio sempre più importante categorie sociali come gli studenti e gli intellettuali. II giornalista Agostino Bisegna ${ }^{4}$ attribuiva proprio a questi ultimi la caduta di Novotný incapace di usoffocare la rivendicazione di una più ampia libertà di espressione sostenuta dagli intellettuali. Costoro, in tutti questi anni, non si sono lasciati irretire né dalle lusinghe, né dalle minaccel» (Bisegna, 6 gennaio 1968: 3). Nessuno della nomenclatura si era reso conto ad esempio di quanto stesse diventando centrale la battaglia degli scrittori che a più riprese avevano denunciato la censura, l'autoritarismo del governo, i crimini compiuti negli anni Cinquanta dal potere comunista. Nel '63 a Bratislava durante un congresso degli scrittori (del quale si ricordava il notevole e coraggioso discorso del poeta Laco Novomeský) e lo stesso anno durante quello dei giornalisti questi temi erano stati posti con inequivocabile chiarezza:

Oggi la stessa opinione pubblica pur operando entro il cerchio di una ferrea dittatura, sta cominciando ad avere partita vinta nei confronti degli ultimi epigoni dello stalinismo come dimostra l'allontanamento di Novotný dalla carica di segretario del partito comunista (Bisegna, 6 gennaio 1968: 3).

La svolta politica poteva inoltre avere rilevanti conseguenze politiche internazionali: dimostrava che «alcuni paesi dell'est si avvicinino alle politiche di

\footnotetext{
${ }^{4}$ Giornalista, studioso e conoscitore del mondo cattolico e di quello comunista ha scritto opere su Pio XII, il Pci e il mondo comunista. Si veda: Bisegna, 1966, 1972.
} 
Kiesingern (II Popolo, 6 gennaio 1968: 1). Domenico Sassoli5, che per il quotidiano democristiano seguì come inviato queste vicende, rimase colpito dallo scarso clamore suscitato presso l'opinione pubblica dalla caduta di Novotný, sottolineando anch'esso l'importanza degli intellettuali ma allo stesso tempo i limiti del nuovo corso. La stampa rimaneva sottoposta a una rigida censura, il dibattito pubblico ridotto all'osso e imprigionato presso una stretta cerchia di intellettuali, impossibilitato ad allargarsi a tutta l'opinione pubblica e la minaccia sovietica incombeva minacciosa (Sassoli, 10 gennaio 1968: 1).

Si cercava anche di comprendere le ragioni profonde degli sconvolgimenti politici cecoslovacchi: perché proprio a Praga? Sassoli, in un successivo articolo del 15 gennaio, giudicava evidente il logoramento del sistema politico comunista imputabile soprattutto all'azione degli «apparati burocratici e di partito dell'unico Paese comunista che segni un arretramento economico rispetto all'anteguerra» (Sassoli, 15 gennaio 1968: 1). La Cecoslovacchia veniva definito il paese upiù frustrato e triste tra quelli dell'estı e tra i suoi mali venivano annoverati il prevalere dell'ideologia sull'economia e l'eccessiva burocratizzazione. Queste vicende politiche erano anche l'occasione per conoscere meglio il paese e raccontarne le peculiarità. Sempre II Popolo ripercorreva i legami culturali tra la Cecoslovacchia e l'Italia andando a ritroso nel tempo. Si partiva dal boom vacanziero dei cecoslovacchi che nel 1967 avevano affollato in 30 mila le spiagge italiane per arrivare ai tempi dell'impero austro-ungarico, quando nella famigerata fortezza dello Spielberg di Brno upatrioti moravi, boemi e italiani hanno stretto tra torture e sofferenze una grande amicizia», senza tralasciare i tragici anni della deportazione di molti cecoslovacchi da parte della Germania nazista per svolgere lavori forzati. Venivano ricordati anche i molti contatti culturali tra i due Paesi (molto apprezzati risultavano Goldoni, Pirandello, Verdi, Rossini) e quelli economici, definiti decisamente felici. Si ricordava, in proposito, anche l'allora recente costruzione di un «grosso e modernissimo stabilimento petrolchimico» da parte dell'Eni a Bratislava (II Popolo, 20 gennaio 1968: 5). II 21 febbraio i capi dei partiti comunisti dell'Europa orientale, esclusi quelli di Jugoslavia e Albania, si incontrarono per un «improvviso verticell a Praga presentato ufficialmente come una celebrazione commemorativa del ventennale dell' ascesa al potere dei comunisti in Cecoslovacchia, avvenuta nel febbraio del 1948. Per Ettore Pettab, del Corriere della Sera, la ricorrenza celebrata «senza rulli di tamburi» forniva ancora una volta chiare indicazioni sul nuovo corso che la segreteria politica di Dubček stava aprendo: «il nuovo capo del partito non sembra propenso a celebrare con troppo clamore la ricorrenza di un avvenimento che ricorda la totale sottomissione della Cecoslovacchia all'Unione Sovietica» (Petta, 22 febbraio 1968: 18).

Venivano forniti anche dettagli politici attenenti le tensioni che permeavano il mondo comunista: il vertice di Praga precedeva quello che si sarebbe tenuto a

\footnotetext{
5 Giornalista, ha scritto di politica estera per La Nazione e II Popolo: https://www.ispionline.it/it/ pubblicazione/chi-e-david-sassoli-il-nuovo-presidente-del-parlamento-europeo-24397 [03/02/2020].

6 È stato per anni il corrispondente per il Corriere della Sera da Vienna, per il quale aveva iniziato a collaborare negli anni '50 da Bonn. Dal 1995 al 1998, anno della sua morte, ha lavorato sempre da Vienna per il quotidiano trentino l'Adige. http://wwwl.adnkronos.com/Archivio/AdnAgenzia/1998/07/ 22/Cronaca/GIORNALISTI-E-MORTO-A-VIENNA-ETTORE-PETTA_164500.php [02/02/2020].
} 
Budapest il 26 febbraio, dove l'Urss temeva di veder emergere una forte critica da parte di Romania e Cecoslovacchia verso la propria leadership, non volendo queste esprimere una chiara condanna nei confronti del maoismo e rinnegando quindi in questo modo, almeno in parte, il ruolo guida sovietico. II giorno seguente l'Avanti! commentava il vertice segnalando la prudenza con la quale si era mosso Brèžnev, costretto a giocare sulla difensiva e a tener conto delle posizioni degli altri partiti comunisti sulla questione cinese (Avanti!, 23 febbraio 1968: 7). I discorsi per celebrare i vent'anni del comunismo in Cecoslovacchia sembravano confermare che Mosca stava rinunciando a condannare le tesi di Pechino e vedeva indebolirsi la sua leadership sul movimento comunista mondiale. Si segnalava anche la posizione di Dubček che giocando la partita cinese cercava allo stesso tempo anche di ribadire la propria linea progressista.

\section{2. uUna svolta a Pragan: il tramonto dei moderati tra le proteste degli studenti}

Tra marzo e aprile il processo di democratizzazione riuscì a mettere a segno altri punti a proprio favore, prima portando al definitivo allontanamento dalla politica di Novotný, che come visto dovette abbandonare anche la carica di Capo dello Stato, e poi assistendo all'approvazione da parte del Comitato centrale del PC cecoslovacco del programma d'azione stilato dal gruppo riformista. All'inizio di marzo una serie di agitazioni studentesche si registrò nelle principali città polacche: Varsavia, Cracovia, Danzica, Lodz e Breslavia. Le manifestazioni più veementi furono quelle di Varsavia dove migliaia di studenti protestarono contro il regime, chiesero decise riforme, denunciarono l'arretratezza e l'autoritarismo del sistema vigente e dovettero fronteggiare la reazione violenta delle forze di polizia". II Messaggero parlò di "sanguinosi scontri per le vie di Varsavia» di «bombe lacrimogene, carri blindati e reparti armati» usati dalla polizia «per reprimere brutalmente una massiccia dimostrazione di studenti ai quali si sono uniti anche operai e impiegati» (II Messaggero, 12 marzo 1968: 1). Le proteste furono innescate dalla sospensione dello spettacolo Dziady, opera dello scrittore romantico Adam Mickiewicz, messo in scena presso il Teatro Nazionale di Varsavia per la regia di Kazimierz Dejmek e sospeso dalle autorità polacche dopo le vibranti proteste dell'ambasciata sovietica che denunciava nella rappresentazione numerosi elementi antirussi. In seguito a questi avvenimenti due esponenti di spicco del movimento studentesco, Adam Michnik e Henryk Szlajfer, che avevano inscenato manifestazioni davanti al monumento di Mickiewicz, vennero espulsi dall'Università di Varsavia (Fik, 1995: 35-103; Eisler, 1968: 146-163; Guida, 2015: 211).

I fatti polacchi venivano collegati con quelli cecoslovacchi: «il fascino che emana quanto avvenuto a Praga è certamente una delle spinte più forti che stanno dietro alla ribellione di Varsaviaı. II 13 marzo il quotidiano romano tornò sulle proteste studentesche che stavano scuotendo il mondo comunista e si cimentò in un ostico parallelo tra le proteste delle università polacche e quelle italiane, finalizzate ad esprimere una forte critica nei confronti del Pci:

[...] ci sarebbe da proporre lo scambio degli studenti tra l'Italia e la Polonia. II fatto è però che la vocazione comunista dei nostri atenei è una pura invenzione del Pci, intenzionato a monopolizzare e anche a rimestare nella medesima pentola tutti gli spunti di malcontento: università, pensionati

\footnotetext{
7 Su queste vicende: Bauman, 2018.
} 
dell'Inps, terremotati siciliani, ex combattenti, per proporsi è chiaro come giustiziere universale (II Messaggero, 13 marzo 1968: 1).

Alla base delle proteste italiane, secondo l'articolo, ci sarebbe stata «una crisi di senescenza dell'istruzione superiore» aggravata "dalle incrostazioni burocratico amministrative del sistema con tutti gli abusi che un tale centralismo autoritario comporta». La vita universitaria è da rivedere ma "lavorando su una maggiore selezione delle capacità più dura di quanto non accada nelle attuali fabbriche dei diplomi di laurea».

In quei giorni anche gli studenti cecoslovacchi si guadagnarono le prime pagine dei giornali italiani recandosi in migliaia sulla tomba di Jan Masaryk. Figlio del primo presidente della Cecoslovacchia Tomáš Garrigue Masaryk, Jan, aveva a lungo ricoperto il ruolo di ambasciatore a Londra (1921-38) e successivamente quello di ministro degli Esteri, prima per il governo cecoslovacco in esilio e successivamente (1945) per il nuovo governo del suo paese. Dopo il colpo di Stato del 1948 fu confermato alla sua vecchia carica, ma morì poco dopo in circostanze oscure ${ }^{8}$. Era chiaro il valore che si dava adesso a queste manifestazioni commemorative, una condanna senza appello da parte dei giovani cecoslovacchi al clima intimidatorio e criminale del vecchio regime. II Popolo spiegava che: «bollato dagli stalinisti come nemico del popolo oggi per molti studenti che lo hanno scoperto di recente è un simbolo di libertàı (II Popolo, 12 marzo 1968: 3).

II 15 marzo il quotidiano democristiano tornava sulle proteste in atto in Polonia e Cecoslovacchia con un editoriale del proprio direttore, Marcello Gilmozzi`, che cercava di cogliere analogie e differenze tra i due fenomeni inquadrandoli però in un generale contesto di crisi del mondo comunista sovietico. Se infatti c'era senza dubbio «un legame tra il crescente fermento di liberalizzazione della Cecoslovacchia e la grande improvvisa fiammata delle dimostrazioni studentesche in Polonia» che dimostrava che "le vecchie strutture comuniste sono incapaci di contenere la nuova realtà sociologica e a una irreversibile avanzata civile») (Gilmozzi, 15 marzo 1968: 1) era anche vero che i due paesi stavano recependo in modo diverso così forti fenomeni. A Praga la classe dirigente «cerca di legittimarsi nei nuovi fermenti invece a Varsavia li si contrastano». In definitiva, i fatti di Praga dimostravano «che la storia non può essere fermata».

Anche il Corriere della Sera con un editoriale di Augusto Guerriero ${ }^{10}$, ripercorreva le vicende cecoslovacche e polacche parlando apertamente di una lenta ma inesorabile "dissoluzione dell'impero fondato da Stalin» e dovuta essenzialmente a due fattori: «lo spirito di nazionalità e lo spirito di libertà» (Guerriero, 18 marzo 1968: 1). Le due proteste erano figlie dello stesso disagio, ma mentre quella polacca si consumava fuori dal partito, quella di Praga era maturata proprio all'interno del

8 Per un profilo biografico: http://www.treccani.it/enciclopedia/jan-masaryk_\%28Dizionario-diStoria\%29/ [02/02/2020].

9 Diventato giornalista professionista nel 1952, ricoprì nel 1958 la carica di direttore del quotidiano L'Adige. In seguito, Aldo Moro lo volle alla direzione del quotidiano II Popolo, che resse per 17 anni fino al 1992. http://wwwl.adnkronos.com/Archivio/AdnAgenzia/2005/12/30/Cronaca/GIORNALISTI-MORTOA-TRENTO-MARCELLO-GILMOZZI-DIRESSE-LADIGE-E-IL-POPOLO_194035.php [02/02/2020].

10 Per un suo profilo: http://www.treccani.it/enciclopedia/augusto-guerriero_(Dizionario-Biografico)/ [02/02/2020]. 
sistema comunista cecoslovacco e si proponeva di riformare il paese mantenendolo in una ben precisa griglia ideologica.

Le notizie arrivavano da Praga a ritmo serrato. I quotidiani italiani ricostruivano con resoconti particolareggiati le rapide trasformazioni della politica cecoslovacca che sembravano poter aver grandi ripercussioni anche su quella internazionale: la lotta di potere all'interno del partito, l'allontanamento di Novotný, il tramonto dei conservatori e l'emergere e il rafforzarsi dei riformisti, le proteste degli studenti, le prime stizzite reazioni da Mosca. II 14 marzo il generale Vladimir Janko, viceministro della Difesa, si tolse la vita perché coinvolto, insieme al generale Jan Sejna, fuggito poi negli Stati Uniti, in un tentativo di colpo di stato a favore di Novotný (Corriere della Sera, 15 marzo 1968: 1).

Il giornalista e studioso di diritto internazionale Ferdinando Vegas ${ }^{11}$, su La Stampa, non aveva dubbi: il mancato golpe segnava il definitivo tramonto politico degli stalinisti. Ci si interrogava ora sulla tenuta della nuova fase:
Al momento sembra che possa evolvere positivamente purché i nuovi dirigenti rimuovano le ultime isole di resistenza e continuino ad appoggiare il movimento rinnovatore, il quale dal canto suo non ripudia il socialismo, ma vuole la democrazia nel socialismo (Vegas, 15 marzo 1968: 1).

Il giorno seguente sempre su La Stampa, venivano descritti sogni, speranze e ambizioni dei giovani cecoslovacchi che speravano non solo di riformare il comunismo e di ritagliarsi nuovi e più ampi margini di libertà, ma anche e soprattutto di arrivare a vivere in un paese che ammettesse completamente una piena pluralità politica:

Forse le speranze della gente sono eccessive su questo punto, in quanto nessun partito comunista ha mai abdicato al potere di propria volontà. Sembra certo però che alla feroce dittatura di Novotný seguirà un regime di tolleranza sull'esempio della Jugoslavia (Conti, 16 marzo 1968: 5).

Il Corriere della Sera spiegava che la stampa russa continuava ad ignorare le vicende cecoslovacche e che i vertici del Cremlino in segno di sfida avevano reso omaggio a Gottwald, il leader comunista autore del colpo di stato a Praga del 1948 (Corriere della Sera, 16 marzo 1968: 1). La tensione iniziava a salire in modo preoccupante, ma la mancanza di un numero sufficiente di soldati sovietici vicino Praga rendeva impossibile una risposta militare, almeno in quel frangente.

Anche Ennio Caretto'2, corrispondente da Mosca per La Stampa, condivideva questa linea. Da una parte si evidenziava una crisi profondissima dello scacchiere comunista in quanto ormai i comunisti di altri paesi dissentivano su qualsiasi questione da Mosca: funzione Onu, Terzo Mondo, non proliferazione nucleare, rapporti con l'Occidente. Di fatto uil blocco non è più quello di una volta e alla luce degli attuali avvenimenti sembra che cambierà in futuro ancora più rapidamente

\footnotetext{
11 A lungo collaboratore de La Stampa (dagli anni '50 fino alla morte avvenuta nel 1984) è stato un autorevole studioso di politica internazionale e docente universitario di storia americana presso I'università di Pavia. Ha diretto I'Ispi (Istituto per gli studi di politica internazionale). Per un ritratto di Ferdinando Vegas: Venturi, 10 maggio 1984: 3.

12 Giornalista ha lavorato a lungo per La Stampa e il Corriere della Sera di cui è stato corrispondente da Washington fino al 2011. Si è occupato di politica americana e di recente di welfare state: Caretto: 2011, 2012, 2013.
} 
senza che l'Unione Sovietica possa farci moltoly (Caretto, 17 marzo 1968: 9). Ma venivano lasciate poche possibilità all'eventualità di un intervento militare: «l'atmosfera ricorda quella del '56 con la differenza che Breznev e Kossighin non possono né vogliono mandare più carri armati in nessuna capitale. Per la prima volta il comunismo sovietico è sulla difensiva» (Caretto, 17 marzo 1968: 9).

Un'articolata lettura dei fatti praghesi veniva fornita dal leader del partito socialista Pietro Nenni proprio su Avanti!: dietro gli eventi in corso c'era un lungo, lento, faticoso, e contrastato processo di critica sotterranea che aveva logorato non soltanto alcuni uomini ma anche alcuni dei dogmi e dei miti alla base della società comunista. Nenni metteva a fuoco anche il problema dell'incompatibilità tra marxismo e violazione dei diritti civili e politici parlando della:

[...]consapevolezza che il balzo socialista dell'umanità dal regno della necessità a quello della libertà, come diceva Marx, comporta il pieno sviluppo della personalità umana attraverso l'integrale esercizio della libertà [...] occorre organizzare lo Stato sulla base della libera articolazione del dissenso a tutti i livelli della vita civile. Oggi siamo a Praga alla prima fase della svolta: si parla, ed è già molto (Nenni, 31 marzo 1968: 1).

La sfida di Praga quindi, in questa lettura si configurava in termini politici come «espressione della grande battaglia dell'umanesimo socialista in tutta I'Europa, in tutto il mondoı. Dal 21 al 31 marzo queste vicende ebbero ampia eco sui quotidiani italiani mentre ad inizio aprile lasciarono per qualche giorno spazio a resoconti e analisi incentrate sul Vietnam, dove i bombardamenti vennero momentaneamente sospesi dall'esercito americano, e sulla decisione del presidente statunitense Lyndon Johnson di non ricandidarsi alle elezioni presidenziali previste per quell'anno.

Al centro dell'attenzione c'erano soprattutto i cambi al vertice dello stato, le rovinose cadute, le rapide ascese, l'emergere di nuove rivendicazioni. Le analisi però convergevano tutte su due punti: il nuovo corso di Dubček reggeva alla reazione dei conservatori e anzi riusciva a strutturarsi in modo sempre più convincente; Mosca aveva rinunciato ad una risposta diretta e violenta optando su una più morbida tesa ad accompagnare e condizionare gli sviluppi politici di questa nuova fase.

II 30 marzo l'ex generale Ludvík Svoboda fu nominato presidente della Repubblica in sostituzione di Novotný. La Stampa lo presentò come "uomo dei russi, protetto da Mosca". Svoboda aveva 75 anni, con il grado di generale durante la seconda guerra mondiale aveva organizzato e guidato una compagnia di partigiani cecoslovacchi che aveva affiancato i sovietici e dopo la guerra aveva ricoperto l'incarico di ministro della Difesa per subire poi all'inizio degli anni Cinquanta «le purghe staliniane» e cadere in disgrazia. Veniva adesso riabilitato e la sua personalità emergeva come figura di compromesso in grado di rassicurare le gerarchie sovietiche e di riequilibrare in parte il peso politico dei progressisti. II quotidiano torinese spiegava che la sua nomina era «stata suggerita dai sovietici a Dubček» e accolta dalle vibranti proteste di «circa seimila universitari» che gridavano per le strade di Praga "abbasso i militari, dateci la democrazia» (Conti, 29 marzo 1968: 15). Anche lo scrittore e inviato speciale del Corriere della Sera Gianfranco 
Piazzesi ${ }^{13}$ considerava Svoboda come un uomo della conservazione e leggeva la sua elezione come un riposizionamento tattico di Dubček desideroso di non aggravare una situazione che già si presentava particolarmente tesa (Piazzesi, 29 marzo 1968: 7). Il leader cecoslovacco cercò di tranquillizzare Mosca ribadendo la fiducia e la fedeltà del suo popolo a quello sovietico e mettendo dei limiti alle riforme varate dal nuovo corso. Lo notava il cronista Massimo Conti su La Stampa:

La nuova democrazia socialista in Cecoslovacchia sarà molto diversa dai modelli dell'Occidente. La guida del Paese resterà nelle mani dei comunisti, gli altri partiti rappresentati al Parlamento di Praga continueranno a svolgere come in passato un programma comune in accordo con le direttive del regime (Conti, 2 aprile 1968: 9).

Dubček in sostanza, definiva i limiti del nuovo corso raffreddando così, le speranze in radicali mutamenti del regime:

\begin{abstract}
Le riforme proposte da Dubček si riducono in sostanza ad una maggiore dialettica delle forze che operano all'interno del sistema: sindacati, categorie sociali ed altri istituti del regime avranno maggiore indipendenza; entro certi limiti la critica sarà possibile anzi incoraggiata. Ma alla aperta ondata polemica che ha accompagnato la caduta di Novotný verranno poste delle remore (Conti, 2 aprile 1968: 9).
\end{abstract}

Proprio in quei giorni il gruppo dei riformisti varò un documento che fatto salvo l'impegno a collaborare con l'Urss, esaltava i principi della sovranità nazionale, dell'indipendenza e dell'uguaglianza, chiamando governo e partito ad assumere un ruolo più attivo nella politica internazionale. La censura preventiva della stampa non veniva abolita e nulla veniva detto sul pieno diritto allo sciopero e sul ruolo della polizia politica. II programma però, affermava «nuovi principi che rappresentano un inizio di legalità dopo vent'anni di feroce dittatura. Ed è importante a parte la loro concreta applicazione che essi siano stati enunciati» (Conti, 10 aprile 1968: 9).

\title{
3. "Da Budapest a Praga»: I'invasione militare e la repressione sovietica
}

Tra il 5 e il 7 maggio, il segretario del Pci Luigi Longo si recò in visita a Praga, dove incontrò i vertici del partito, rilasciò interviste al quotidiano del regime Rude Pravo ed espresse la vicinanza dei comunisti italiani al gruppo dei riformatori. L'Unità, presentando la visita di Longo, spiegò che il colloquio tra questo e Dubček si era consumato in un'atmosfera "cordiale e fraterna» e si sottolineava anche che il leader progressista «confermava che il processo di democratizzazione» non sarebbe stato "sfruttato in senso antisocialista» (Goruppi, 7 maggio 1968: 1). Longo poi, esprimeva un giudizio ampiamente favorevole sui cambiamenti in atto: «l'esperienza cecoslovacca servirà ai Pc dei paesi capitalistici nella battaglia per creare una società socialista giovane, aperta e moderna». L'Unità glissava sulle divergenze tra Praga e Mosca, che apparivano nei fatti ancora profonde, non approfondiva i nodi critici della svolta progressista e presentava una situazione che sembrava essersi pienamente ricomposta.

Eppure il 10 maggio le ombre di un possibile e tragico intervento armato sovietico si materializzarono nuovamente all'orizzonte a causa di alcune manovre militari

13 Giornalista e scrittore, Piazzesi ha collaborato tra gli altri con La Nazione, La Stampa, il Corriere della Sera. Per un suo ritratto: Pieroni, 26 gennaio 2001: 37. 
sovietiche nei pressi dei confini cecoslovacchi. Il Corriere d'Informazione aprì la sua edizione con una sola domanda: "a Praga come a Budapest?» (Petta, 10 maggio 1968: 1). Il Popolo parlò di «drammatiche notizie provenienti dalle ambasciate occidentali di Varsavia» e si chiese se un intervento militare fosse imminente. Grande spazio ebbero anche un drammatico appello lanciato da Radio Praga (uper amor di Dio che non si ripetano per noi la dolorosa esperienza della Jugoslavia o addirittura gli avvenimenti di Budapest. Lasciateci assumere la responsabilità del nostro futuro. Sappiamo ciò che vogliamo e dove andiamoı) e un improvviso vertice politico tenutosi a Mosca tra i leader sovietici del partito (II Popolo, 10 maggio 1968: 1). Franco Gerardi, il direttore di Avanti!, sul quotidiano socialista parlò espressamente di una «tragica realtàı:
Il drammatico appello di Radio Praga ha aperto uno squarcio nel velo che ancora circonda la effettiva situazione esistente nell'Europa comunista: ed è una realtà tragica e terribile quella che vi si vede in fondo, la realtà di paesi che non sono tuttora liberi di scegliere le proprie strade e il proprio destino, la realtà di popoli civilissimi ai quali la via dell'emancipazione e la lotta per la verità sono sbarrate dal rischio, dal terrore dell'intervento e dell'oppressione straniera. Esprimiamo innanzitutto la nostra solidarietà col popolo cecoslovacco: manifestiamo la speranza che tutto si fermi all'angoscia di queste ore e che una nuova ondata di buio e di lutto sia evitata all'Europa e al mondo: alziamo forte la nostra voce di vomini liberi, assieme a quella di tutti i socialisti e di tutti i democratici di ogni paese, per condannare, questo ennesimo attentato alla pace e allo sviluppo pacifico dei popoli (Gerardi, 10 maggio 1968: 1).

Un allarme contro il quale il Pci rispose piuttosto duramente attraverso il proprio segretario, Luigi Longo che accusò il quotidiano socialista di uestremismo, settarismo, faziositàı in quanto impegnato ad estremizzare la situazione politica in Cecoslovacchia (Avanti!, 14 maggio 1968: 1). La situazione però sembrò schiarirsi quasi subito e la maggior parte degli osservatori continuò a ritenere improbabile una escalation militare. Nicola Caracciolo ${ }^{14}$ su La Stampa analizzando i rapporti tra Washington e Mosca osservava che:

\begin{abstract}
I russi hanno fatto sapere agli americani di non contemplare la possibilità di interventi armati allo stato attuale delle cose. I movimenti di truppe alla frontiera andavano intesi più come un intervento ai cecoslovacchi a non spingersi troppo lontano, che come una precisa minaccia. Gli americani dal canto loro hanno fatto sapere ai russi di non avere nessuna intenzione di incoraggiare i cecoslovacchi a mettersi su una strada che avrebbe potuto ad esempio portarli fuori dal Patto di Varsavia (Caracciolo, 14 maggio 1968: 18).
\end{abstract}

Venivano anche sottolineati, dall'Avanti!, i progressi compiuti dal nuovo corso: riforme economiche e più ampie libertà di stampa, annunciate come imminenti entro il mese di giugno (Avanti!, 17 maggio 1968: 1). Queste vicende accendevano anche la curiosità della stampa italiana sulla produzione cinematografica e letteraria della Cecoslovacchia. Un momento per fare il punto della situazione fu rappresentato dal meeting del cinema organizzato dall'azienda autonoma di Alghero col patrocinio della Regione Sardegna, una sei giorni di proiezioni di film in

\footnotetext{
14 Giornalista, studioso di storia contemporanea e autore televisivo, Nicola Caracciolo è stato a lungo corrispondente per La Stampa da Washington. Per un suo profilo biografico: Caracciolo, 1986.
} 
concorso e fuori concorso che prevedeva anche una mostra dedicata ai libri dai quali erano stati tratti i film in rassegna ${ }^{15}$. II 26 giugno il Corriere della Sera dedicò un articolo allo scrittore cecoslovacco Bohumil Hrabal, ospite ad Alghero insieme ad Alberto Moravia e Dacia Maraini, dove venne proiettato un film di Jiři Menzel Treni strettamente sorvegliati vincitore del premio Oscar come miglior film straniero nel 1966 e tratto da una sua opera omonima del 1965. II quotidiano italiano spiegava che Hrabal unon ha avuto alcuna difficoltà a raccontare le peripezie degli scrittori cecoslovacchi durante gli anni duri del regime» e che per molto tempo "era addirittura inutile andare dagli editori, tanto di questioni che sfiorassero non solo la politica ma la vita del paese, non volevano parlarney) (Corriere della Sera, 26 giugno 1968: 11). Durante la rassegna furono presentate diverse pellicole cecoslovacche, le dominatrici non a caso della manifestazione, che fu pensata in quella edizione proprio come un omaggio e un segno di vicinanza al Paese: «si può dire che Alghero abbia voluto rendere un omaggio particolare alle lucide energie dei cineasti di Praga che hanno sviluppato in questi ultimi anni nuove e coraggiose ragioni di creazione artistica» (Corriere della Sera, 1 luglio 1968: 11).

Oltre a l'Estate capricciosa, sempre di Menzel e II quinto cavaliere è la paura di Zbyněk Brynych venne presentato anche II segno del Cancro (in Italia tradotto come Assassinio a sangue caldo). II Corriere della Sera nel recensirlo notava che:

La struttura è di un film poliziesco, ambientato in un grande ospedale di Praga, dove un medico è stato trovato assassinato in circostanze misteriose. Ma in realtà il racconto prevarica subito la sua impostazione apparente, per assumere i toni di un'aspra requisitoria politica tra le più decise e coraggiose che siano state tentate in Cecoslovacchia, prima della recente svolta democratica (Corriere della Sera, 29 giugno 1968: 13).

La Cecoslovacchia fu protagonista anche a Rimini nell'ambito della rassegna del cinema per la gioventù che si tenne nella città romagnola dall' 1 al 6 luglio. L'evento fu seguito interamente da La Stampa e prevedeva due momenti: nel pomeriggio, film per ragazzi, di sera, lungometraggi sulla problematica della gioventù (La Stampa, 4 luglio 1968: 7). Non mancavano riferimenti mondani: la giovanissima attrice Andrea Čunderlíková, diciassettenne, protagonista della pellicola Piccolo blues d'estate, veniva immortalata in costume sulla spiaggia di Rimini (La Stampa, 6 luglio 1968: 7). La Cunderlikova veniva definita «una quasi diva acqua e sapone che indossa la minigonna e non conosce Kafka»l né si interessava di politica e delle proteste degli studenti e chiedeva invece il permesso alla mamma per partecipare a qualsiasi attività mondana. Per La Stampa, "questa Gigliola Cinquetti prima maniera, curiosa ed ingenua» rappresentava il volto innocente e spensierato di un paese lontano ma ormai perennemente presente nel dibattito pubblico internazionale (La Stampa Sera, 6 luglio 1968: 3). Proprio in quei giorni nuove manovre militari dell'esercito sovietico tornarono a far temere il peggio. Secondo Ferdinando Vegas de La Stampa «l'atmosfera tra Praga e Mosca si offusca gravemente» e sulla Cecoslovacchia tornava ad allungarsi «l'ombra sanguinosa di Budapest» (Vegas, 11 luglio 1968: 1). Il paragone era ormai esplicito con la situazione esistente in Ungheria nell'ottobre del 1956, «dopo che Breznev, pochi giorni or sono, aveva fatto un richiamo sin troppo trasparentels. Tuttavia la maggioranza degli

15 L'evento si svolse dal 23 al 28 giugno e vide la presenza in concorso di otto pellicole (La Stampa, 24 giugno 1968: 7). 
opinionisti e degli esperti del mondo comunista continuava a ritenere remota la possibilità di un intervento armato di Mosca come accaduto dodici anni prima a Budapest, a iniziare dallo stesso Vegas:

I due casi sono assai diversi: allora I'Ungheria era uscita dal Patto di Varsavia, aveva restaurato il pluripartitismo, stava scivolando fuori dal socialismo; oggi, invece, i dirigenti cechi hanno dato ai sovietici, le più ampie ed aperte assicurazioni che non intendono abbandonare il Patto di Varsavia e che sono decisi a mantenere il monopolio del partito comunista. Breznev e compagni sanno benissimo che Dubček, Smrkovsky, Cisar e gli altri attuali capi cecoslovacchi non sono affatto degli estremisti del movimento di liberalizzazione; sono invece dei progressisti moderati, che cercano di mantenersi in equilibrio tra le opposte pressioni, in una difficile lotta su due fronti (Vegas, 11 luglio 1968: 1).

La nuova dirigenza comunista di Dubček aveva avanzato riforme caparbie e coraggiose ma con calcolata prudenza, senza uscire dal campo socialista, garantendo la propria vicinanza al patto di Varsavia e all'Urss. Si tratta di un'analisi che tornerà spesso nei giorni dell'invasione. Anche secondo lo scrittore e giornalista Carlo Casalogno ${ }^{16}$ lo scopo principale delle minacce sovietiche era quello di "esercitare un'energica pressione su Praga" (Casalogno, 15 luglio 1968: 1). Da più parti veniva esaltato, comunque, il coraggio del popolo cecoslovacco. Enzo Bettiza $^{17}$ ad esempio sul Corriere d'Informazione osservava che la

\begin{abstract}
Cecoslovacchia sta affrontando una prova di resistenza con una grande potenza aggressiva [...] è tutta una piccola nazione che sta vivendo le sue ore col fiato sospeso. I commenti della gente, gli interventi della radio, le note liberissime della radio, le note liberissime della stampa, seguono di ora in ora le lentissime e contorte operazioni di falso spostamento delle truppe sovietiche che non sembrano uscire dal paese (Bettiza, 23 luglio 1968: 2).
\end{abstract}

Gianfranco Piazzesi sul Corriere della Sera dedicava un lungo ritratto a Ludwig Vaculic, "sscrittore ardito e saggio che dopo aver acceso le polveri ha indicato ai politici la formula nuova. Questo scrittore è divenuto l'vomo di punta degli innovatori». Secondo Piazzesi era "arrivato il momento della verità. I russi premono e per la prima volta minacciano di reprimere [...] è arduo prevedere come i cecoslovacchi si comporteranno se sapranno resistere oppure se piegheranno la testa» (Piazzesi, 20 luglio 1968: 5). La notte tra il 20 e il 21 agosto, le truppe sovietiche supportate da una coalizione di Paesi alleati comprendenti Polonia, Repubblica Democratica Tedesca, Ungheria e Bulgaria invasero la Cecoslovacchia. Nei giorni successivi Dubček e altri esponenti di primo piano del governo cecoslovacco furono portati a Mosca dove dovettero accettare la rinuncia a ogni disegno riformatore e il ritorno su posizioni conservatrici filosovietiche. L'intervento militare vide la partecipazione di 350.000 soldati sovietici, di reparti speciali dell'intelligence militare, il GRU, e di 80.000 soldati del Patto di Varsavia per un totale di 500.000 unità (Bottoni,

\footnotetext{
16 Partigiano e membro del Partito d'Azione, Casalegno entrò a La Stampa nel 1947 per diventarne vicedirettore nel 1968. Coraggioso e tenace nemico del terrorismo di matrice politica che imperversava in quel periodo in Italia, fu assassinato da un commando delle Brigate Rosse il 29 novembre del 1977. Sulla sua vicenda professionale e umana: Casalegno, 2008; Marletti \& Bullo, 2004.

17 Giornalista, scrittore e politico italiano ha lavorato nel corso della sua carriera per La Stampa, Corriere della Sera e Il Giornale. È stato senatore ed eurodeputato con il Partito Liberale (1976-1989) e con il Partito Socialista italiano (1989-1993). Per un suo ritratto: Cazullo, 2017: 46.
} 
2011: 219). Questa prova di forza, nelle intenzioni di Mosca, avrebbe dovuto testimoniare anche l'unità del mondo comunista nel reprimere la svolta progressista di Praga. Ma in realtà, fu seguito da più di una lacerazione: il 21 agosto il leader comunista romeno Ceaușescu condannò apertamente l'intervento (Bottoni, 2011: 219). Una tragica manifestazione di dissenso arrivò da Varsavia a settembre, quando Ryszard Siwiec, un ex soldato dell'Armata Nazionale (Armia Krajowa), si dette fuoco, uccidendosi, per esprimere solidarietà con il popolo cecoslovacco (Blažek, 2010).

L'operazione militare condotta da Mosca fu comunque rapida e colse di sorpresa l'opinione pubblica internazionale, compresa la stampa italiana.

Quest'ultima sottolineò soprattutto la brutalità dell'aggressione, lo sdegno sollevato in tutto il mondo dalla violenta operazione sovietica, la condanna senza appello avanzata da istituzioni e partiti politici e, infine, gli accostamenti con l'Ungheria. Molte analisi cercarono di spiegare come mai la possibilità di un intervento armato fosse stata scartata in modo così clamorosamente errato da opinionisti ed esperti di politica internazionale che ben conoscevano contesti e scenari in questione. Indro Montanelli18, sul Corriere della Sera, riprese l'analisi proposta qualche settimana prima da Ferdinando Vegas su La Stampa: la sorpresa era data dalla radicale differenza dello scenario tra la Budapest del 1956 e la Praga del 1968. Qui i riformisti non si erano compromessi in azioni eccessivamente rischiose ai danni dei russi, non avevano ecceduto con la piazza, si erano mossi in modo lucido e accorto conquistando il partito, mettendo all'angolo gli esponenti più conservatori, isolando le forze fedeli a Mosca, conquistando un ampio consenso presso l'opinione pubblica e giurando fedeltà al Comecon e al patto di Varsavia. D'altro canto, anche i sovietici sembravano cambiati, più aperti ai cambiamenti: «ancora una volta ci siamo sbagliati, ancora una volta il nostro pessimismo sul regime sovietico peccava di ottimismol» (Montanelli, 22 agosto 1968: 3). L'intervento secondo Montanelli era anche peggiore di quello del 1956: a Budapest i sovietici si erano accollati interamente la responsabilità politica dell'invasione, stavolta avevano coinvolto altre truppe del patto di Varsavia e prima dell'attacco a Cierna e Bratislava avevano firmato nuovi accordi con Praga riconoscendo politicamente i cambiamenti e operando, quindi, uno squallido doppio gioco. Venivano mosse anche delle critiche al Pci: secondo Montanelli i comunisti italiani si erano dissociati dall'attacco militare in modo assai mite, autorizzati dalla stessa Mosca per evitare futuri, possibili rovesci elettorali.

Proprio l'organo del Pci, l'Unità, riportando il "grave annuncio da Praga" parlò di "emozione e profonda preoccupazione nel movimento operaio internazionale" (l'Unità, 21 agosto 1968: 1), sottolineando distinguo e dissensi nei confronti dell'intervento all'interno dello stesso mondo comunista. In particolare, venne sottolineata la posizione critica di Ceacescu, di Tito e dei partiti comunisti occidentali come quelli francese e italiano (I'Unità, 22 agosto 1968: 1).

Piero Ottone'19 sul Corriere d'Informazione riteneva l'intervento sovietico un gravissimo errore tattico da parte di Mosca che andava a soffocare il tentativo attuato da Dubček di «conciliare la libertà con il comunismo»:

\footnotetext{
18 Su Indro Montanelli: http://www.treccani.it/enciclopedia/indro-montanelli/ [31/01/2020].

19 Su Pietro Ottone: http://www.treccani.it/enciclopedia/ricerca/piero-ottone/ [30/01/2020].
} 
[...] in caso di successo il suo esempio avrebbe acquisito una forza irresistibile di irradiazione. I movimenti riformisti nel blocco orientale si sarebbero rinvigoriti e le resistenze della conservazione sarebbero state travolte [...] l'esperimento di Dubček poteva quindi mutare l'aspetto del comunismo internazionale ma i conservatori russi sono convinti che un regime comunista non può reggersi sul consenso popolare (Ottone, 22 agosto 1968: 1).

Il giornalista e scrittore Panfilo Gentile, sul Corriere della Sera, spiegava l'errore di valutazione della stampa italiana con l'insensatezza dell'operazione militare:

L'invasione della Cecoslovacchia è del tutto sproporzionata e folle se si guarda quel che era in gioco e cioè l'innocente desiderio di Praga di fare in casa propria un po' di comodo proprio senza danni di nessuno, diventa una tragedia per chi come l'Unione sovietica ha visto nell'assaggio liberale cecoslovacco il fallimento totale dei propri dogmi [...] il romeno Ceaușescu è uscito indenne, pur avendo assunto atteggiamenti assai più arroganti, perché non era un eretico (Gentile, 22 agosto 1968: 1).

Era stata l'eresia, dunque, a risultare inaccettabile per il socialismo reale, monolitico e inscalfibile di Mosca:

Dubček è invece un eretico sia pure innocuo e l'Unione Sovietica ha avuto paura dell'eresia e del propagarsi dell'eresia. E la paura fa perdere la testa. Per l'Unione Sovietica questa invasione [...] è una battaglia perduta (Gentile, 22 agosto 1968: 1).

II saggista e intellettuale Alberto Ronchey20 leggeva le ragioni profonde dell'aggressione in una intrinseca debolezza del regime sovietico: «forse i capi russi hanno temuto il contagio dei fermenti di libertà nel blocco sovietico: anche nell'Urss si manifestano inquietanti segni di dissensol) (Ronchey, 22 agosto 1968: 3). Un fatto però sembrava certo: «la grande macchina del prestigio dell'Urss è da oggi in un lungo tunnel, dal quale sarà difficile uscirem. Non c'era spazio solo per la politica. Igor Man che seguì l'occupazione militare da Praga, scrisse un resoconto drammatico e vibrante delle ore tragiche che si stavano consumando. Arrivato fortunosamente in città dopo un lungo viaggio da Vienna, Man racconta di una città spettrale, sospesa in un clima di terrore e attesa, ancora sotto choc:

Scrivo in fretta, convulsamente, interrompendomi di tanto in tanto per correre alla finestra: il cielo, gonfio di nuvole che hanno cancellato le stelle, è solcato a tratti da proiettili traccianti. Improvvisamente un bengala allaga l'oscurità di luce sinistra e bagliori d'incendio illuminano gli antichi campanili, le torri barocche. S'apre un greve silenzio, subito rotto dallo spalare degli elicotteri, quindi dalle strade sale il crepitare rabbioso dei fucili mitragliatori. Gli elicotteri cercano di individuare gli automezzi su cui si spostano i trasmettitori di Radio Libertà; le pattuglie dei mongoli dell'Armata rossa, del para bulgari sparano contro i giovani che, obbedendo agli ordini del Fronte della Resistenza, si affannano a svellere i numeri civici, a cancellare i nomi delle strade per fuorviare la polizia segreta sovietica a caccia di patrioti (Man, 25 agosto 1968: 3).

Arrivavano anche storie e racconti da parte dei turisti italiani sorpresi a Praga dal precipitare degli eventi. L'ambasciata italiana gli avviò alla frontiera cerando di

20 Per un profilo biografico: http://www.treccani.it/enciclopedia/alberto-ronchey [29/01/2020]. 
gestire le operazioni nel modo più ordinato possibile. II Corriere della Sera raccontò la storia di due turisti milanesi: al loro seguito anche «due belle ragazze ceche che hanno scelto la libertàı. Si trattava di due studentesse di Praga, che arrivate a Milano, chiesero il permesso di soggiorno sperando di trovare lavoro e alloggio nel capolvogo lombardo (Corriere della Sera, 26 agosto 1968: 4). La tragedia cecoslovacca e la battaglia dei riformisti di Dubček suggerirono al giornalista e scrittore Alberto Jacometti una riflessione sulla stampa indipendente che:

[...]rappresenta una voce libera, che non serviva nessun padrone se non gli interessi dei lavoratori e del popolo. Sono assolutamente convinto che una delle paure dell'Unione Sovietica sia stata: la possibilità della prevalenza di un partito che non fosse il comunista, conseguenza della nascita di una pluralità di partiti, conseguenza a sua volta della libertà di opinione[...] la censura è di fatto la fine della democrazia e della libertà (Jacometti, 25 agosto 1968: 2).

Lo scrittore Ugo Leonizio su Avanti!, nella rubrica del giovedì «inchieste e cronache culturaliz descrisse lo spirito combattivo, tenace ma allo stesso tempo composto dei cecoslovacchi come molto simile a quello del buon soldato Sc'vèik, il militare inetto e imbranato, fortunato protagonista dei racconti antimilitaristi di Jaroslav Hašek. L'inno di Sc'veik «ci vuole calma» è una "attitudine che si compendia in forma di ironia, filosofia (nell'accezione di prendersela con filosofia) saggezza» e lo "«svejkismo è risorto una volta di più durante questi giorni che hanno tramutato la "primavera praghese" in un duro inverno. Forse proprio quest'ironia rurale, tipicamente ceca della creatura di Hasek, ha evitato un Vietnam nel centro Europa» (Leonizio, 29 agosto 1968: 3). La Primavera di Praga era all'epilogo. Le principali riforme furono abrogate e Dubček fu dapprima isolato politicamente e poi, nell'aprile del 1969, sostituito alla guida del partito comunista cecoslovacco da Gustav Husak. Quell'anno, dal 16 gennaio al 24 aprile un gruppo di giovani si suicidò dandosi fuoco in luoghi pubblici al fine di manifestare la propria indignazione per l'epilogo autoritario che la stagione di riforme avviata dal gruppo riformista aveva avuto. II primo fu uno studente di 21 anni, Jan Palach. Per La Stampa Sera era il simbolo più drammatico ed evidente del "calvario di un popolo che non vuole arrendersi» (La Stampa Sera, 21 gennaio 1969: 1). Nel settembre del 1969 il mensile comunista II Manifesto, con un intervento di Lucio Magri, commentava amaramente:

La Cecoslovacchia non suscita più vera emozione. I leaders del nuovo corso restano, nella migliore delle ipotesi, dei simboli; non più esponenti di una forza o di una linea. La resistenza di base, disperata quanto tenace, obbliga ai tempi lunghi, alla espressione negativa, come passività e sabotaggio, può finir col perdere il suo carattere socialista. Che si può ragionevolmente attendere dal domani, se non che sia più grave dell'oggi? (Magri, 1969: 1).

\section{Conclusione}

La Primavera di Praga è stato uno degli avvenimenti spartiacque del secondo dopoguerra. Dopo aver avviato un'articolata fase di riforme e tentato di ritagliarsi uno spazio politico autonomo e originale nello scacchiere politico dell'Europa comunista, il progetto politico di Dubček si arenò travolto dalla stretta autoritaria di Mosca. Per molti osservatori, si trattò di una vicenda dalla chiarissima valenza 
simbolica, che mostrava il vero volto dell'Europa a trazione sovietica e quanto fosse irriformabile quel sistema. Quindi

\begin{abstract}
La Primavera di Praga costituì in tal modo, secondo Francesco Leoncini, l'ultima grande utopia socialista e democratica del Novecento. II celebre filosofo marxista ungherese György Lukács, costretto a un'umiliante autocritica dopo il 1956 per evitare l'espulsione dal partito, confessò a un amico che con gli eventi cecoslovacchi era "probabilmente" fallito l'intero esperimento iniziato nel 1917 (Bottoni, 2011: 222).
\end{abstract}

Il progetto riformista cecoslovacco, che aveva sollevato molte illusioni e speranze, godette di una grande simpatia presso l'opinione pubblica italiana. La stampa nazionale, infatti, seguì da vicino le principali fasi dell'esperienza politica di Dubček sottolineando sin da subito il tramonto politico di dirigenti di estrazione stalinista come Novotný e l'emergere di una nuova classe dirigente ambiziosa e decisa a sollevare dei nodi politici rimasti per troppo tempo irrisolti. Successivamente venne prestata grande attenzione al ruolo ricoperto nelle trasformazioni cecoslovacche da intellettuali e studenti, cercando, anche, di cogliere analogie e differenze con quanto stava accadendo nelle università italiane. Infine, osservatori e cronisti registrarono amaramente, la brutale repressione sovietica che chiudeva l'esperimento di Praga e mostrava in modo palese i limiti e le criticità di un sistema che dietro la promessa di una alleanza democratica e fraterna tra i vari paesi socialisti, strangolava in realtà qualsiasi tentativo di divincolarsi dal controllo di Mosca. Molti quotidiani nei mesi precedenti l'intervento armato dell'Urss, avevano sottolineato l'accortezza strategica dei riformisti cecoslovacchi e ritenevano improbabile una prova di forza di Breznev. La solidarietà espressa dalla stampa italiana al popolo cecoslovacco e la condanna decisa alla svolta militare, con l'eccezione de l'Unità che maturò una critica più sfumata, si unirono di pari passi a una constatazione che trovò quasi all'unanimità spazio sulle principali testate nazionali: I'inconciliabilità tra comunismo sovietico e libertà e la grave crisi di quel sistema politico.

\title{
Bibliografia
}

(5 gennaio 1968). Antony Novotný defenestrato dalla carica di primo segretario. Il Messaggero, 4, p. 1.

(6 gennaio 1968). Imbarazzo di Pankov per la caduta di Novotný. II Popolo, 5, p. 1.

(7 gennaio 1968). Praga: il problema di fondo è la democratizzazione. Avanti!, 6, p. 3.

(7 gennaio 1968). Un mutamento radicale nel regime cecoslovacco. II Popolo, 6, p. 1.

(20 gennaio 1968). Praga fa l'appello all'Italia per l'economia e la cultura. II Popolo, 19 , p. 5.

(23 febbraio 1968). Insolitamente moderato Brezhnev verso Pechino. Avanti!, 47, p. 7.

(12 marzo 1968). Omaggio ufficiale alla tomba di Masaryk. II Popolo, 61, p. 3.

(12 marzo 1968). Un omaggio ai ribelli di Praga. Corriere della Sera, 150, p. 11. 
(12 marzo 1968). Violenta requisitoria a Praga contro i privilegi dei dirigenti comunisti. Il Messaggero, 71, 1968, p. 1.

(13 marzo 1968). Gli studenti di Varsavia. Il Messaggero, 72, p. 1.

(15 marzo 1968). Suicida a Praga un vice-ministro sospettato di complotto stalinista. Corriere della Sera, 63, p. 1.

(16 marzo 1968). I russi onorano Gottwald in contrasto con il nuovo corso ceco. Il Corriere della Sera, 64, p. 1.

(10 maggio 1968). Praga a Mosca: non ripetete la tragica esperienza ungherese. II Popolo, p.111, p. 1.

(14 maggio 1968). II Pci condannato dai fatti. Avanti!, 113, p. 1.

(17 maggio 1968). A Praga libertà di stampa e nuove riforme economiche. Avanti!, 116, p. 1.

(26 giugno 1968). Da Praga la voce del dissenso. Corriere della Sera, 146, p. 11.

(29 giugno 1968). Sorprese e conferme nei film cecoslovacchi. Corriere della Sera, 149 , p. 13.

(4 luglio 1968). La Cecoslovacchia protagonista a Rimini alla rassegna del cinema per la gioventù. La Stampa, 153, p. 7.

(6 luglio 1968). Da Praga al sole di Rimini. La Stampa, 155, p. 7.

(6 luglio 1968). Piomba a Rimini una quasi diva all'acqua e sapone. La Stampa Sera, 156, p. 3.

(22 agosto 1968). Ore drammatiche a Praga. I'Unità, 222, p. 1.

(22 agosto 1968). Truppe sovietiche entrano in Cecoslovacchia. I'Unità, 221, p. 1.

(26 agosto 1968). Due belle ragazze ceche hanno scelto la libertà. Corriere della Sera, 194, p. 4.

(21 gennaio 1969). II calvario d'un popolo che non vuole arrendersi. La Stampa Sera, 17, p. 1.

AJtMATOV, C. (1992). Il giorno che durò più di un secolo. Milano: Mursia.

Arbatov, G. (1992). The system. An Insider's Life in Soviet politics. New York: Times Books.

BAUMAN, Z. (2018). Gli si diceva...Varsavia 1968. Roma: Castelvecchi.

BetTIZA, E. (23 luglio 1968). Un popolo in ansia. Corriere d'Informazione, 164, p. 2.

- (2009). La primavera di Praga: 1968, la rivoluzione dimenticata. Milano: Mondadori.

BIAGI, E. (20 marzo 1968). Novotný, l'ultimo protagonista del buio dopoguerra staliniano. La Stampa, 68, p. 3.

BISEGNA, A. (6 gennaio 1968). La caduta di Novotný. II Popolo, 5, p. 3.

- (1966). Pio XII nel vortice della tempesta. Stato sociale. Rivista mensile di studi finanziari economici sociali, 10 (3). 
- (1972). Vennero da lontano... il Pci dal 1944 ad oggi. Roma: Accademia romana di sociologia.

BLAžEK, P. (2015). Ryszard Siwiec 1909-1968. Instytut Pamieci Narodowej: Praga.

BoffA, G. (6 gennaio 1968). Dubček sostituisce Novotný come segretario del partito. I'Unità, 5, p. 12.

BotTonI, S. (2011). Un altro Novecento. L'Europa orientale dal 1919 a oggi. Roma: Carocci.

Caracciolo, N. (14 maggio 1968). Mosca assicura gli Stati Uniti che non interverrà a Praga: esclusi interventi militari. La Stampa, 114, p. 18.

CARETTO, E. (17 marzo 1968). Mosca inquieta sui fatti di Varsavia e Praga: non c'è più in Europa un impero sovietico. La Stampa, 66, p. 9.

- (2011). Le due torri: i dieci anni che hanno sconvolto l'America. Roma: Editori Internazionali Riuniti.

- (2013). Quando l'America si innamorò di Mussolini. Roma: Editori Riuniti.

CASAlegno, A. (2008). L'attentato. Milano: Chiarelettere.

CASAlogno, C. (15 luglio 1968). Pesante minaccia. Stampa Sera, 160, p. 1.

CAzzullo, A. (29 luglio 2017). II lungo esilio di un intellettuale mittleuropeo. Corriere della Sera, 178, p. 46.

CONTI, M. (16 marzo 1968). Silurato un ministro, un altro sotto accusa a Praga per il fallito putsch dei militari. La Stampa, 65, p. 5.

- (29 marzo 1968). Gli studenti di Praga protestano per la scelta di un uomo de russi a capo dello Stato. La Stampa, 76, p. 15.

- (2 aprile 1968). Praga avrà maggiore libertà ma nei limiti del regime comunista. La Stampa, 79, p. 3.

- (10 aprile 1968). Praga annuncia il distacco dall'Urss ed auspica rapporti con Bonn e l'Occidente. La Stampa, 86, p. 9.

DANIEL, J. (1977). II diritto di dire no in Libertà e socialismo. Momenti storici del dissenso. Milano: SugarCo.

DI Giorgio, M. (2020). II giornale-partito: per una storia del Manifesto. Roma: Odradek.

Dicorato, G. (2012). Andavamo al Giamaica. Personaggi e storie anche politicamente scorrette di mezzo secolo di giornalismo a Milano. Bologna: Lampi di Stampa.

EISLER, J. (1991). Marzec 1968: geneza, przebieg, konsekwencje. Varsavia: Państwowe Wydawnictwo Naukowe.

FIK, M. (1995). Marcowa kultura. Varsavia: Wydawnictwo Wodnika.

Fornaro, P. \& Fedel, S. (ed.) (2009). La Primavera di Praga. Quarant'anni dopo. Soveria Mannelli: Rubbettino.

Gentile, P. (22 agosto 1968). La paura dell'eresia. Corriere della Sera, 191, p. 1. 
GerardI, F. (10 maggio 1968). Un angoscioso appello di Radio Praga a Mosca: In nome di Dio, non ripetiamo il dramma ungherese, Avanti!, 110, p. 1.

GILMOzzl, M. (15 marzo 1968). Da Praga a Varsavia: la crisi del sistema. II Popolo, 74, $\mathrm{p} 1$.

GORUPPI, S. (7 maggio 1968). Dubček: i compagni sovietici hanno compreso i nostri sforzi. l'Unità, 124, p. 1.

GrazIOSI, A. (2008). L'Urss dal trionfo al degrado. Storia dell'Unione Sovietica. 19451991. Bologna: II Mulino.

Guerriero, A. (18 marzo 1968). Gli studenti polacchi. Corriere della Sera, 66, p. 1.

GUIDA, F. (2009), Era sbocciata la libertà? A quarant'anni dalla Primavera di Praga (1968-2008). Roma: Carocci.

- (2015). L'altra metà dell'Europa. Roma-Bari: Laterza.

HAVEL, V. (1991). Il potere dei senza potere. Milano: Garzanti.

Hobel, A. (2001). II Pci, il '68 cecoslovacco e il rapporto col PCUS. Studi storici, 4, pp. $1145-1172$.

JACOMETtI, A. (25 agosto 1968). La funzione del giornale. Avanti!, 194, p. 2.

Laudiero, A. (2018). La Primavera di Praga e le sue stagioni: storia e storie. Roma: Viella.

LeONCINI, F. (1998). Primavera di Praga. Storia e dossier, 126, pp. 12-21.

- (2003). L'Europa centrale. Conflittualità e progetto. Passato e presente tra Praga, Budapest e Varsavia. Venezia: Libreria Editrice Cafoscarina.

- (ed.). (1989). Alexander Dubček e Jan Palach. Protagonisti della storia europea. Soveria Manelli: Rubbettino.

- (ed.). (2007). Che cosa fu la Primavera di Praga? Idee e progetti di una riforma politica e sociale. Venezia: Libreria Editrice Cafoscarina.

LEONIZIO, U. (29 agosto 1968). L'unità della cultura cecoslovacca nella lotta per il nuovo corso. Avanti!, 197, p. 3.

MAN, I. (25 agosto 1968). Tra l'indomita folla di Praga. La Stampa, 192, p. 3.

MAGRI, L. (1969). Praga è sola. Il Manifesto, 4, p. 1.

Marletti, C. (2004). II Piemonte e Torino alla prova del terrorismo. Soveria Manelli: Rubbettino.

Montanelli, I. (22 agosto 1968). Da Budapest a Praga. Corriere della Sera, 191, p. 3.

NenNI, P. (31 marzo 1968). La svolta di Praga. Avanti!, 77, p. 1.

OTtONE, P. (22 agosto 1968). Una vittoria apparente. Corriere d'Informazione, 188, p. 1.

Pajetta, G. (1982). Le crisi che ho vissuto. Budapest, Praga, Varsavia. Roma: Editori Riuniti. 
PetTA, E. (22 febbraio 1968). Improvviso vertice comunista a Praga. Corriere della Sera, 45, p. 18.

- (10 maggio 1968). A Praga come a Budapest? Corriere d'Informazione, 109, p. 1.

PIAZZESI, G. (29 marzo 1968). Era legato allo stalinismo il successore designato di Novotný, Corriere della Sera, 75, p. 7.

- (20 luglio 1968). La coscienza di Praga. Corriere della Sera, 165, p. 5.

PieronI, A. (26 gennaio 2001). Piazzesi, il romanziere del compromesso storico. Corriere della Sera, 126, p. 36.

Ripellino, A. M. (2008). L'ora di Praga. Scritti sul dissenso e sulla repressione in Cecoslovacchia e nell'Europa dell'Est (1963-1973). Firenze: Le lettere.

RONCHEY, A. (22 agosto 1968). Perché la brutale aggressione? La Stampa, 189, p. 3.

RozgonovÀ, D. (2009). Una testimonianza sulla Primavera di Praga e sui giorni dell'occupazione sovietica. In Fedele, S. \& Fornaro, P. (ed.). La Primavera di Praga quarant'anni dopo. Soveria Manelli: Rubbettino, pp. 5-13.

SASSOLI, D. (10 gennaio 1968). Le drammatiche fasi della caduta di Novotný. II Popolo, 21, p. 1.

- (15 gennaio 1968). L'industria cecoslovacca in ritardo di vent'anni. II Popolo, 25, p. 1.

Sterpellone, A. (7 gennaio 1968). Se ne va lo stalinista Novolny ma il Cremlino controlla sempre Praga. II Messaggero, 7, p. 1.

VEGAS, F. (15 marzo 1968). Una svolta a Praga. La Stampa, 64, p. 1.

- (11 luglio 1968). Sulla Cecoslovacchia l'ombra sanguinosa di Budapest. La Stampa, 161, p. 1.

VentuRI, A. (10 maggio 1984). Studioso da 30 anni alla Stampa. La Stampa, 110, p. 3.

VolCiC, D. (2008). L'autunno di Praga. Palermo: Sellerio.

ZASLAVSKY, V. (2009). La Primavera di Praga: resistenza e resa dei comunisti italiani. In FEDEle, S. \& FornARO, P. (ed.). La Primavera di Praga quarant'anni dopo. Soveria Manelli: Rubbettino, pp. 99-119. 